\title{
Studies on the Kinetics of Muon Catalyzed Fusion in the HT Mixture with Very Low Tritium Concentration
}

\author{
S. Nasrin Hoseinimotlagh, Sakineh Ghaderi, and Fatemeh Mozafari K.
}

\begin{abstract}
A method for determination of optimum muon cycling coefficient and energy gain in the HT mixture with very low tritium concentration is proposed. The kinetics of the mu-atomic and mu-molecular processes preceding the pt reaction in the $p t \mu$ molecule is described. The time variations of $\gamma$ quanta and conversion muons and the other particles formation in nuclear fusion reactions in $p t \mu$ molecules are studied. Our calculations show that, the optimum value of muon cycling coefficient at $C_{t}=0.01$ is equal to 106 . In this paper our obtained results from theoretical calculations and experimental results are compared with together and we can receive that the obtained results are in good agreement with measured values.
\end{abstract}

Index Terms-Muon cycling, HT mixture, mu-atomic, mu-molecular, fusion.

\section{INTRODUCTION}

The idea of muon catalyzed fusion $(\mu C F)$ was first suggested by C.Frank in 1947, [1] when he tried to explain tracks from cosmic rays in photo emulsions exposed at high altitudes .Although his explanations was not correct (the tracks were in reality positive muons from pion decays at rest).The experimental discovery of $\mu C F$ was achieved at the end of 1956 in Berekely by L.W.Alvarez team looking at bubble chamber pictures [2]. A muon $(\mu)$ is a leptonic elementary particle and has a finite lifetime of $2.2 \mu \mathrm{s}$. Since the mass of a muon 207 times larger than an electron, the size of an exotic atom/molecule containing the negative muon is much smaller than an electronic atom/molecule. When the negative muon binds to hydrogenic nuclei (proton, $\mathrm{p}$, deuteron, d, or triton, t) like $H_{2}^{+}$, a nuclear fusion reactions occurs in the muonic molecular ion, for example :

$$
(d t \mu)^{+} \rightarrow{ }^{4} \mathrm{He}+n+\mu^{-}
$$

The muon does not take part in the nuclear reaction directly but only catalyzes the reaction. This process is called muon catalyzed fusion. The pt reaction is one pf the least known of all processes of $\mu_{C F}$ in the mixture of hydrogen isotopes.

It is very important to gain information on reaction characteristics of all muonic processes in HT mixture (e.g.,

Manuscript received November 9, 2013; revised January 17, 2014. This work was supported in part by the Department of Physics, Shiraz branch Islamic Azad University, Shiraz, Iran.

The authors are with Department of Physics, Shiraz branch Islamic Azad University, Shiraz, Iran (e-mail: hoseinimotlagh@hotmail.com, sakineh.ghaderi.1990@gmail.com,e.mozaffari10@yahoo.com). the rate of muon transfer from $p \mu$ atom to triton, the rate of transition between hyperfine levels of $t \mu$ atoms, the rate of formation of the $p t \mu$ molecule, the rate of nuclear synthesis in it and muon sticking) to interpret correctly the results of experiments in the triple mixture of hydrogen isotopes H-D-T and to describe the kinetics of all processes occurring in the mixture. From the theoretical point of view, the experiments investigating $\mu C F$ processes in hydrogen -tritium mixture will allow one to test an algorithm describing a three-body system of particles interacting according to coulomb rule. It is necessary to emphasize the importance of the $\mu C F$ study in HT mixture in order to obtain the information about characteristics of pt-reaction at ultra law energy range $(\approx K e V)$. The investigation of the reaction between light nuclei at ultra law energies $(\approx K e V)$ is very important for verification of fundamental symmetries in string interactions [3]-[5] the contribution of muon exchange currents and to solve some astrophysical problems. At present, there are few experiments that investigate characteristics of $\mu C F$ in a $\mathrm{H}-\mathrm{T}$ mixture. Only one was performed with a HT mixture and the references with triple mixture H-D-T. In this paper the authors survey the major areas of research: Section I describes the details of the kinetics of the $\mu C F$ in HT mixture, while Sec. II describes the nonlinear point dynamics equations in HT mixture. Sec.III discusses on the numerical solution of these equations versus time in muon life time range. Sec.IV describes the optimum cycling coefficient and energy gain. The final section presents an overview of what is known, and outline some directions for future research.

\section{INTRODUCING OF THE KINETICS OF $\mu C F$ IN HT MIXTURE}

The scheme of $\mu-$ atomic and $\mu$-molecular processes in the HT mixture after the negative muons stopped, is shown in Fig. 1. As a result of muon transfers $p \mu-$ atom to tritium nuclei

$$
p \mu+t \rightarrow t \mu+p+183 e V
$$

$t \mu$ atoms are formed with the kinetic energy of about $45 \mathrm{eV}$ (the scheme in Fig. 1 corresponds to a very low tritium concentration in HT mixture $(\leq 1 \%)$, which allows one to neglect direct capture of the muon by tritium).

The ground state of the $t \mu$ atom is split into two hyperfine structure levels with $F=S_{t}+S_{\mu}$ being the total spin of the $t \mu$ atom $S_{t}=S_{\mu}=\frac{1}{2}$ are the spins of triton and muon, respectively) equal to $F=1\left(\left(S_{t} S_{\mu}\right) \equiv(\uparrow \uparrow)\right)$ and 
$F=0\left(\left(S_{t} S_{\mu}\right) \equiv(\uparrow \downarrow)\right)$. The energy of hyperfine splitting of the $t \mu$ atom equals $0.24 \mathrm{eV}$. The initial population of hyperfine levels is assumed statistically to be: $\eta=\frac{3}{4}(F=1)$, $\eta=\frac{1}{4}(F=0)$.In the collision of $t \mu$ atoms with $H_{2}$ or $H T$ molecules

$$
\begin{gathered}
t \mu+H_{2} \rightarrow\left[(p t \mu)^{+} p e\right]^{+}+e^{-} \\
t \mu+H T \rightarrow\left[(p t \mu)^{+} t e\right]^{+}+e^{-}
\end{gathered}
$$

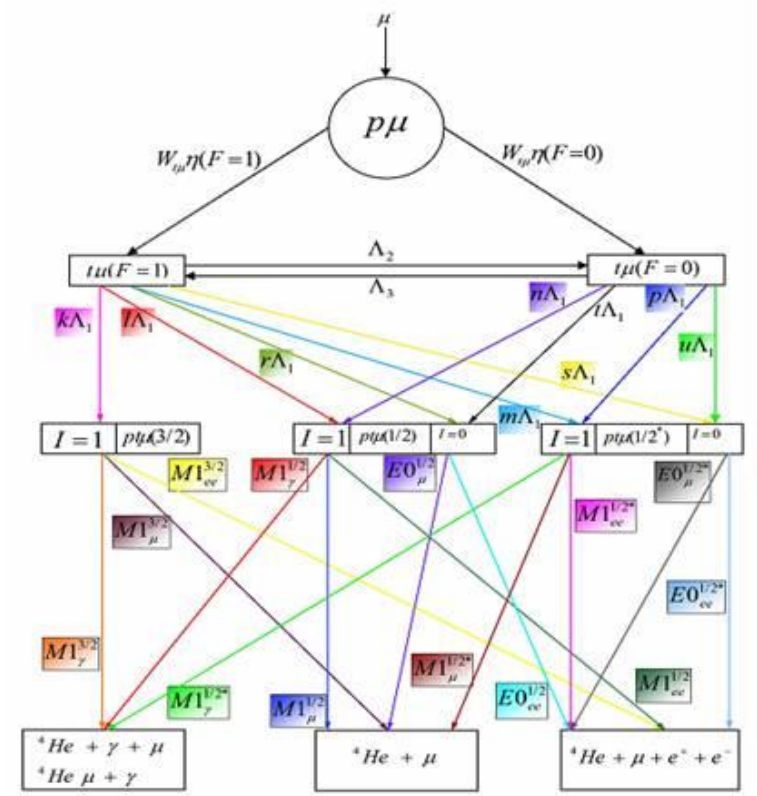

Fig. 1. Comprehensive fusion reaction network in HT mixture with low tritium concentration.

The $p t \mu$ molecule is formed by the electric dipole transition $\mathrm{E} 1$ in excited state $(J, v)$ (where $J, v$ are rotational and vibrational quantum numbers of the $\mathrm{pt}$-system in $p t \mu$ molecule, respectively).At the collision of the $t \mu$ atom with triton in the $T_{2}$ or $H T$ molecule, the formation of a $t t \mu$ molecule is possible

$$
\begin{gathered}
t \mu+T_{2} \rightarrow\left[(t t \mu)^{+} t e\right]^{+}+e^{-} \\
t \mu+H T \rightarrow\left[(t t \mu)^{+} p e\right]^{+}+e^{-}
\end{gathered}
$$

due to $\mathrm{E} 1$ dipole transition. The competitive processes to the formation of a $p t \mu$ molecule are: free muonic decay $\left(\mu \rightarrow e^{-}+v_{\mu}+v_{e}^{-}\right), t t \mu$ molecule formation (processes (5)and (6) and the $t \mu$ atom transition between hyperfine levels.

$$
\begin{gathered}
t \mu(F=1)+t \stackrel{\lambda_{10}}{\longrightarrow} t(F=0)+t \\
t \mu(F=0)+t \stackrel{\lambda_{01}}{\longrightarrow} t(F=1)+t
\end{gathered}
$$

The transition (8) is possible only when the energy of the $t \mu$ atom fulfils the condition:

$\left.E_{t \mu}\right\rangle \Delta E=0.24 \mathrm{eV}$ ( $\Delta E$ is energy of hyperfine splitting of the ground state of the $t \mu$ atom). The probability of the transition of a $t \mu$ atom between hyperfine levels due to the collision of $t \mu$ atom with a proton

$$
t \mu(F=1)+p \underset{\longleftrightarrow}{\stackrel{\lambda_{10}}{\longleftrightarrow}} t \mu(F=0)+p
$$

This is very small [6] (because of the small rate of spin flip reactions due to spin-spin interactions compared to the rate of charge exchange reactions).The transition of $p t \mu$ molecule from the state with $(J v)=(10)$ to the ground state $(J v)=(00)$ proceeds very quickly $\left(\approx 10^{-11} s\right)$ and the energy difference between two states is carried out by conversion electron. The ground state of the $p t \mu$ molecule is split into three sublevels with total momentum $j=I+S=\frac{3}{2}, \frac{1}{2}, \frac{1}{2}^{*}$ [7]

The binding energy of the ground state of the pt $\mu$ molecule (in the non relativistic case) equals $\varepsilon_{00}=214 \mathrm{KeV}$. The probability of the formation of a pt $\mu$ molecule in the state with total momentum $j$ and nuclear spin $I=1$ in the collision of $t \mu$ atom in the orto-state with a proton is smaller than during the collision of $t \mu$ atom in the para- state.The populations of the states with different $j, J, I$ and $S$ ( $\mathrm{S}$ is the total spin of $p t \mu$ molecule) depend on the relations between the rate of the loss of the energy by the $t \mu$ atom (due to elastic and non-elastic collisions with $H_{2}, H T$ and $T_{2}$ molecules), the rates of the processes (7-8) $\left(\lambda_{10}, \lambda_{01}\right)$, and also on the relation between the above mentioned rates and the rate of the $p t \mu$ molecule formation. The whole set of nuclear reactions occurring in the $p t \mu$ molecule in different states:

$$
\begin{array}{r}
\text { pt } \mu \rightarrow{ }^{4} \mathrm{He}+\mu+\gamma+19.76 \mathrm{MeV} \\
p t \mu \rightarrow{ }^{4} \mathrm{He} \mu(1 s)+\gamma+19.77 \mathrm{MeV} \\
\text { pt } \mu \rightarrow{ }^{4} \mathrm{He} \mu(2 p)+\gamma \\
\text { pt } \left.\mu \rightarrow{ }^{4} \mathrm{He} \mu(n\rangle 2\right)+\gamma \\
p t \mu \rightarrow{ }^{4} \mathrm{He}+\mu+19.22 \mathrm{MeV} \\
\text { pt } \mu \rightarrow{ }^{4} \mathrm{He} \mu+e^{+}+e^{-} \\
\text {pt } \mu \rightarrow{ }^{4} \mathrm{He} \mu+\gamma+\gamma
\end{array}
$$

The production of $19.8 \mathrm{MeV} \gamma$ quanta (M1- transition) is 
possible only from the state of $p t \mu$ molecule with the total nuclear spin $I=1$ (reactions $(10 a-10 d)$ ). Non radioactive transitions $(10 e)$ and $(10 f)$ proceed dominantly via the monopole E0 transition. The probability for the reaction channel $(10 \mathrm{~g})$ is negligibly small. The values of the partial rates for the different $p t \mu$ decay channels can be written as:

$$
\lambda_{f, i}^{p t}(j)=\rho\left(W_{j}^{0} K_{0}^{i}+W_{j}^{1} K_{1}^{i}\right)
$$

where $i \equiv \gamma, \mu, e^{+}, e^{-}, 2 \gamma ; \rho$ is the density of the probability that the distance between the proton and triton in the $p t \mu$ molecule equals 0 and $K_{0}^{i}, K_{1}^{i}$ are pt reaction constants for s wave in the nuclear states with $I=0$ (singlet) and $I=1$ (triplet). For the theoretical description of the pt reaction we use the resonant model of the existence of ${ }^{4} \mathrm{He}$ nuclei in excited state $0^{+}$near the threshold of this reaction .It is seen that from Fig. 1 that transitions (7-8) change the populations of the state of the $p t \mu$ molecule (the population of the state with $j=\frac{3}{2}$ decreases, therefore together with the $p t \mu$ molecule formation, the process of thermalization of $t \mu$ atoms proceeds ) which can change not only the yield of the reaction products (10) but also the ratio between the partial probabilities for different channels of the reaction.

\section{DESCRIPTION OF DYNAMICAL EQUATIONS IN THE HT MIXTURE WITH VERY LOW TRITIUM CONCENTRATION}

The point dynamical equations which are the rate equations for various particle comprehensive network reactions (Fig. 1) are written as following:

$$
\begin{aligned}
& \frac{d N_{\mu}}{d t}=S_{\mu}-\lambda_{a} C_{p} \varphi N_{\mu}+\left(1-\omega_{S}\right)\left(M 1_{\gamma}^{3 / 2} N_{p t \mu_{3 / 2}}^{I=1}+M 1_{\gamma}^{1 / 2} N_{p t \mu_{1 / 2}}^{I=1}\right. \\
& +M 1_{\gamma}^{1 / 2^{*}} N_{p t \mu_{1 / 2^{*}}^{I I}}^{I=1}+M 1_{\mu}^{3 / 2} N_{p t \mu_{3 / 2}}^{I=1} \\
& +M 1_{\mu}^{1 / 2} N_{p t \mu_{1 / 2}}^{I=1}+E 0_{\mu}^{1 / 2} N_{p t \mu_{1 / 2}}^{I=0}
\end{aligned}
$$

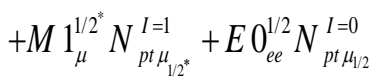

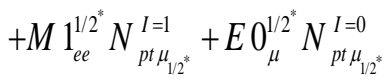

$$
\begin{aligned}
& \left.+M 1_{e e}^{3 / 2} N_{p t \mu_{3 / 2}^{I}}^{I=1}+M 1_{e e}^{1 / 2} N_{p t \mu_{1 / 2}}^{I=1}+E 0_{e e}^{1 / 2^{*}} N_{p t \mu_{1 / 2^{*}}^{I=0}}^{I=}\right) \\
& \frac{d N_{p \mu}}{d t}=\lambda_{a} c_{p} \phi N_{\mu}-W_{t \mu} \eta(F=1) N_{p \mu} \\
& -W_{t \mu} \eta(F=0) N_{p \mu}-\lambda_{0} N_{p \mu} \\
& \frac{d N_{t \mu}^{F=1}}{d t}=W_{t \mu} \eta(F=1) N_{p \mu}+\Lambda_{3} N_{t \mu}^{F=0}-\Lambda_{2} N_{t \mu}^{F=1} \\
& -\left(k \Lambda_{1}+l \lambda_{1}+m \Lambda_{1}+s \Lambda_{1}\right) N_{t \mu}^{F=1}-\lambda_{0} N_{t \mu}^{F=1}
\end{aligned}
$$

$$
\begin{aligned}
& \frac{d N_{p t \mu_{3 / 2}}^{I=1}}{d t}=k \Lambda_{1} N_{t \mu}^{F=1}-M 1_{\gamma}^{3 / 2} N_{p t \mu_{3 / 2}}^{I=1}-M 1_{\mu}^{3 / 2} N_{p t \mu_{3 / 2}}^{I=1} \\
& -M 1_{e e}^{3 / 2} N_{p t \mu_{3 / 2}}^{I=1}-\lambda_{0} N_{p t \mu_{3 / 2}}^{I=1} \\
& \quad \frac{d N_{p t \mu_{1 / 2}}^{I=1}}{d t}=l \Lambda_{1} N_{t \mu}^{F=1}+n \Lambda_{1} N_{t \mu}^{F=0}-M 1_{\gamma}^{1 / 2} N_{p t \mu_{1 / 2}}^{I=1} \\
& -M 1_{\mu}^{1 / 2} N_{p t \mu_{1 / 2}}^{I=1}-M 1_{e e}^{1 / 2} N_{p t \mu_{1 / 2}}^{I=1}-\lambda_{0} N_{p t \mu_{1 / 2}}^{I=1}
\end{aligned}
$$

$$
\begin{aligned}
& \frac{d N_{p t \mu_{1 / 2}}^{I=0}}{d t}=r \Lambda_{1} N_{t \mu}^{F=1}+t \Lambda_{1} N_{t \mu}^{F=0}-E 0_{\mu}^{1 / 2} N_{p t \mu_{1 / 2}}^{I=0} \\
& -E 0_{e e}^{1 / 2} N_{p t \mu_{1 / 2}}^{I=0}-\lambda_{0} N_{p t \mu_{1 / 2}}^{I=0}
\end{aligned}
$$

$$
\begin{aligned}
& \frac{d N_{p t \mu_{1 / 2^{*}}^{I=1}}^{I}}{d t} m \Lambda_{1} N_{t \mu}^{F=1}+p \Lambda_{1} N_{t \mu}^{F=0}-M 1_{\gamma}^{1 / 2^{*}} N_{p t \mu_{1 / 2^{*}}}^{I=1} \\
& -M 1_{\mu}^{1 / 2^{*}} N_{p t \mu_{1 / 2^{*}}}^{I=1}-M 1_{e e}^{1 / 2^{*}} N_{p t \mu_{1 / 2^{*}}^{I=1}-\lambda_{0}}^{I=1} N_{p t \mu_{1 / 2^{*}}^{I=1}}
\end{aligned}
$$$$
\frac{d N_{p t \mu_{1 / 2^{*}}}^{I=0}}{d t}=s \Lambda_{1} N_{t \mu}^{F=1}+u \Lambda_{1} N_{t \mu}^{F=0}-
$$$$
E 0_{e e}^{1 / 2^{*}} N_{p t \mu_{1 / 2^{*}}}^{I=0}-E 0_{\mu}^{1 / 2^{*}} N_{p t \mu_{1 / 2^{*}}^{I=0}}^{I=}-\lambda_{0} N_{p t \mu_{1 / 2^{*}}^{I=0}}^{I=}
$$

where, $N$ are the particle densities for muons, atoms, molecules and $\gamma$ quanta. $\lambda_{0}=0.445 \times 10^{6} s^{-1}$ is the free muon decay rate; $\lambda_{p t}, \lambda_{10}, \lambda_{p t \mu}$ are the rates of the muon transition from $p \mu$ atom to triton, of the transition of $t \mu$ atom from the state with $F=1$ to the state with $F=0$,and of the pt $\mu$ molecule formation, respectively (the above values are reduced to liquid hydrogen density, $\left.n_{0}=4.25 \times 10^{22} \mathrm{~cm}^{-3}\right) ; \lambda_{f, \mu}^{p t}(I=0), \lambda_{f, \mu}^{p t}(I=1)$ are partial rates of nuclear synthesis in the pt $\mu$ molecule with muon production for the total spin of proton and triton equal to 0 and1,respectively; $\lambda_{f, e e}^{p t}(I=0), \lambda_{f, e e}^{p t}(I=1)$ are the rates of nuclear synthesis in the $p t \mu$ molecule with the formation of an electron-positron pair for the total spin of $\mathrm{p}$ and $\mathrm{t}$ equal to 0 and 1, respectively; $C_{p}$ and $C_{t}$ are atomic concentrations of 
protium and tritium in HT mixture; $\phi$ is the density of the $\mathrm{H}-\mathrm{T}$ mixture reduced to liquid hydrogen density, also E0 and M1 are the electric dipole and magnetic dipole transitions rate respectively.

\section{MUON STICKING}

During the fusion reaction, there is a probability that the muon will stick to a charged product. In pt fusion reaction (Eq.(10)) the muon can stick to ${ }^{4} \mathrm{He}$ by forming a muonic helium ion $\left(\mu^{4} \mathrm{He}\right)$.In this case, the muon stays bound to the

${ }^{4} \mathrm{He}$ particle and is therefore lost for the foregoing chain of reactions. The sticking probabilities for pt-fusion reaction is equal to $\omega_{s}=0.95$.

\section{Calculation of Muon Cycling Coefficient}

For a certain volume of fusion reactor core, the protium and tritium are injected. Considering a reactor operating period $\tau$ during which a general muon injection rate $S_{\mu}$ occurs. Then, the total energy supplied and total nuclear energy released as a consequence of injection can be calculated. Using these calculated parameters, the $X_{c}$ which represents the average number of pt fusion events catalyzed by one muon is determined:

$$
X_{c}=\frac{\int_{0}^{\tau+\tau_{\mu}} \lambda_{p t \mu}^{f} N_{p t \mu}(t) d t}{\int_{0}^{\tau} S_{\mu}(t) d t}
$$

where:

$$
\begin{aligned}
& \lambda_{p t \mu}^{f} N_{p t \mu}(t)=\lambda_{p t \mu}^{f, I=1}\left(N_{p t \mu \frac{3}{2}}^{I=1}(t)+N_{p t \mu \frac{1^{*}}{2}}^{I=1}(t)\right)+ \\
& \lambda_{p t \mu}^{f, I=0}\left(\sum_{p t \mu \frac{1}{2}}^{I=0}(t)+N_{p t \mu \frac{1}{2}}^{I=0}(t)\right)
\end{aligned}
$$

and $\tau_{\mu}=2.2 \times 10^{-6} \mathrm{~s}$ is the muon life time. To determine $X_{c}$, we must solve the equations (12-21) in time interval $0 \leq t \leq \tau_{\mu}$.Also the energy gain is defined as: $G=\frac{Q_{p t} X_{c}}{E_{\mu}}$. $Q_{p t}=3.5 \mathrm{MeV}$ is the fusion energy of the pt- reaction and $E_{\mu}=4.5 \mathrm{GeV}$ is the required energy for production of muon.

\section{Numerical CALCUlations}

The numerical values of $X_{c}$ and $\mathrm{G}$ are calculated by using Eq.(23) and (25) for various relative concentration ratio of $C_{t}\left(0 \leq C_{t} \leq 0.01\right)$, and $\phi=1 L H D$ for the available data. The optimum values of $X_{c}$ and $\mathrm{G}$ with selection of $C_{t}=0.01$ are equal to 106 and 0.08, respectively. In Fig. 2 to Fig. 10, the time variation of different particle densities such as $\quad N_{\mu}, N_{p \mu}, \quad N_{t \mu}^{F=1}, N_{t \mu}^{F=0}$, $N_{p t \mu \frac{3}{2}}^{I=1}, N_{p t \mu \frac{1}{2}}^{I=1}, N_{p t \mu \frac{1}{2}}^{I=0}, N_{p t \mu \frac{1}{2}}^{I=0}$ and $N_{\gamma}$ as a function of $C_{t}$ are shown, respectively.

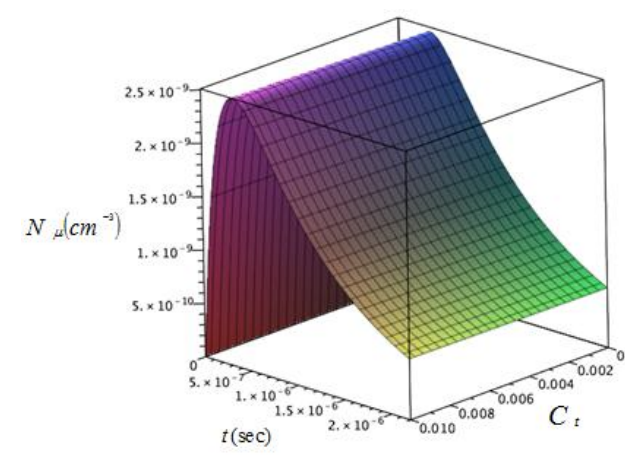

Fig. 2. The time variation of $N_{\mu}$ at time interval $0\left\langle t\left\langle 2.2 \times 10^{-6} s\right.\right.$ as a function of $C_{t}$

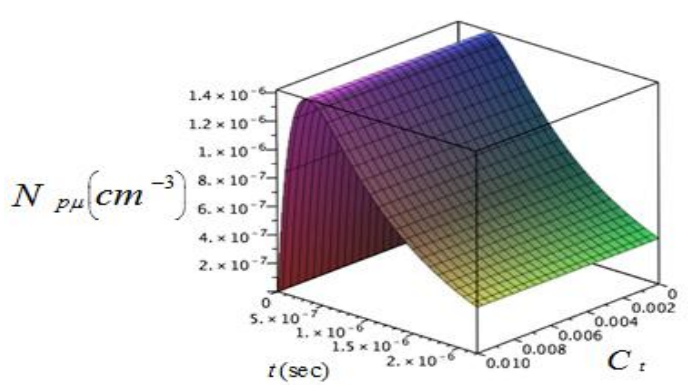

Fig. 3. The time variation of $N_{p \mu}$ at time interval $0\left\langle t\left\langle 2.2 \times 10^{-6} s\right.\right.$ as a function of $C_{t}$

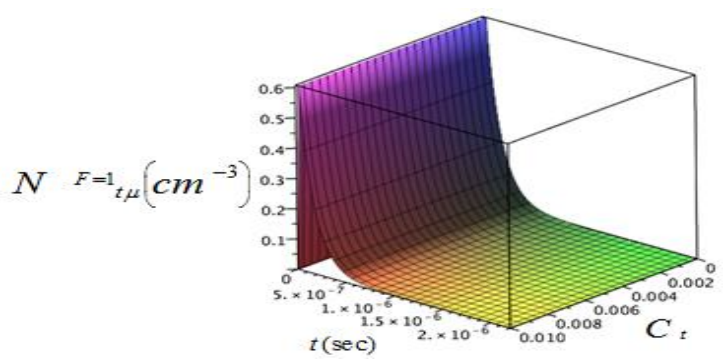

Fig. 4. The time variation of $N_{t \mu}{ }^{F=1}$ at time interval $0\left\langle t\left\langle 2.2 \times 10^{-6} s\right.\right.$ as a function of $C_{t}$

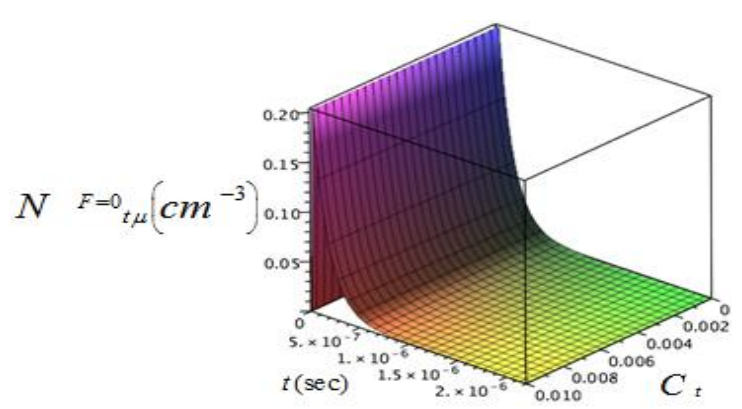

Fig. 5. The time variation of $N_{t \mu}{ }^{F=0}$ at time interval $0\left\langle t\left\langle 2.2 \times 10^{-6} s\right.\right.$ as a function of $C_{t}$. 


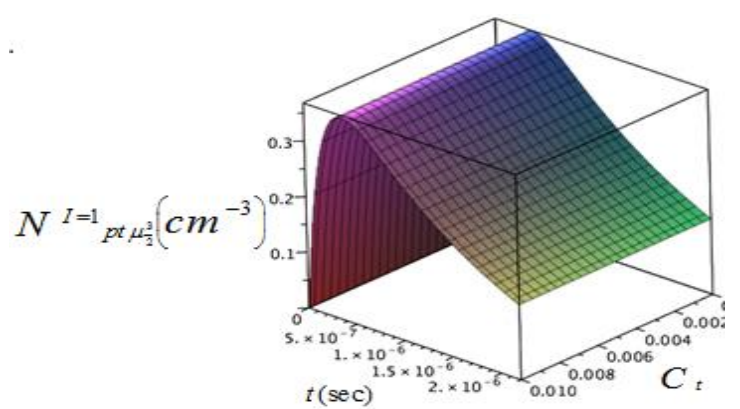

Fig. 6. The time variation of $N_{p t \mu \frac{3}{2}}^{I=1}$ at time interval $0\left\langle t\left\langle 2.2 \times 10^{-6} s\right.\right.$ as a function of $C_{t}$.

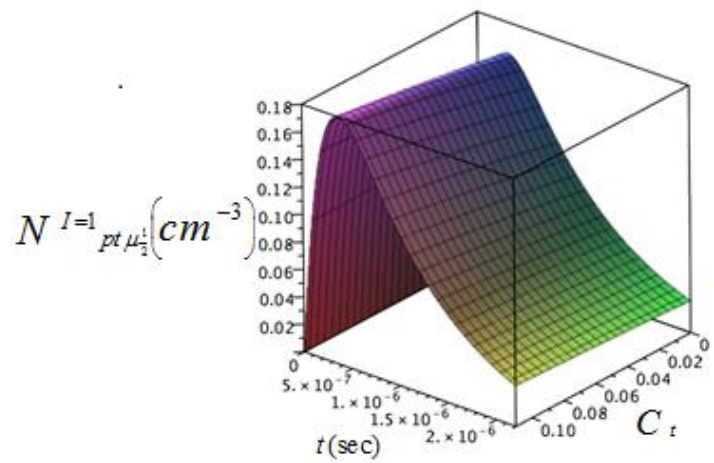

Fig. 7. The time variation of $N_{p t u \frac{1}{2}}^{I=1}$ at time interval $0\left\langle t\left\langle 2.2 \times 10^{-6} s\right.\right.$ as a function of ${ }_{C_{t}}$.

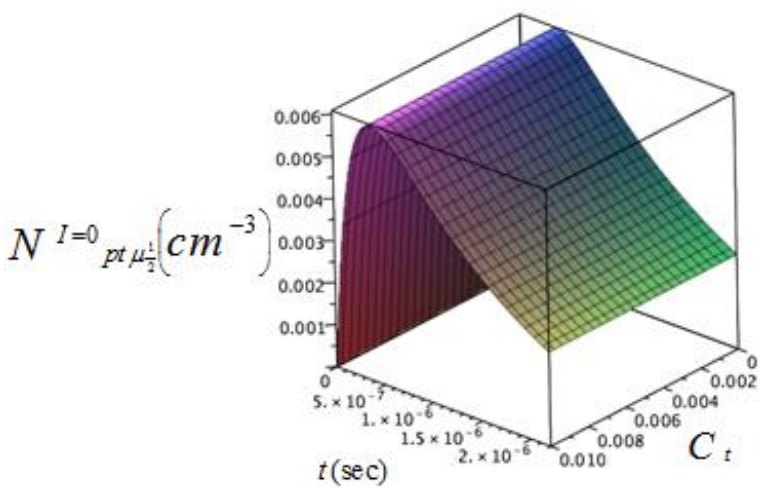

Fig. 8. The time variation of ${ }_{p t u \frac{1}{2}}^{I=0}$ at time interval $0\left\langle t\left\langle 2.2 \times 10^{-6} s\right.\right.$ as a function of $C_{t}$

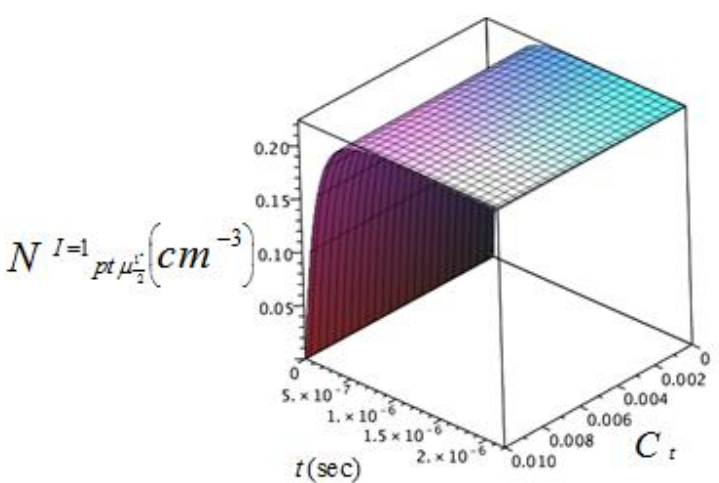

Fig. 9. The time variation of $N_{p t \mu \frac{1}{2}}{ }^{{ }^{*}}{ }^{1=1}$ at time interval $0\left\langle t\left\langle 2.2 \times 10^{-6} s\right.\right.$ as a function of $C_{t}$.

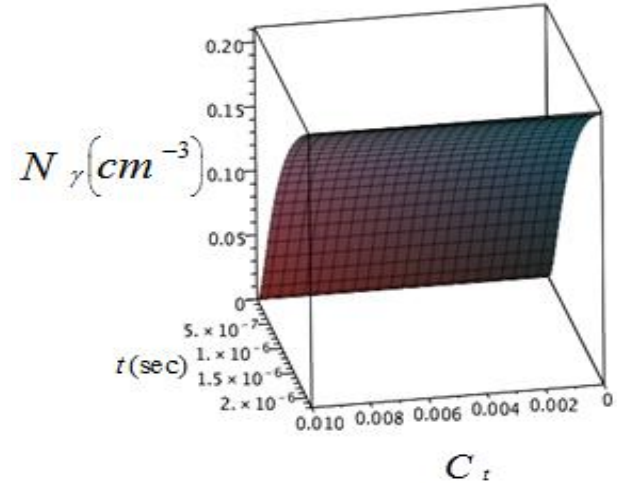

Fig. 10. The time variation of $N_{\gamma}$ at time interval $0\left\langle t<2.2 \times 10^{-6} s\right.$ as a function of $C_{t}$.

\section{CONCLUSION}

From the presented analysis of the $\mu C F$ kinetic in the HT mixture we can conclude that the muon cycling coefficient in $p t \mu$ is increasing respect to muon cycling coefficient of $p t \mu$ branch in HDT mixture, this is due to that in the HDT mixture, the other fusion reaction branches $(\mathrm{dt} \mu, \mathrm{dd} \mu, \mathrm{pd} \mu$, $\mathrm{tt} \mu$ ) are formed, and $d t \mu$ branch has maximum value muon cycling coefficient approximately equal to 111 because the $d t \mu$ molecule has a maximum fusion rate and minimum muon sticking coefficient, but one of the advantage of the selection of HT mixture respect to the HDT mixture is that, for reaching to maximum value cycling rate in HT mixture only we required to tritium concentration $C_{t}=0.01$, but in the HDT mixture tritium concentration is increased. The other advantage of HT mixture is that tritium is radioactive and its production is very expensive. The disadvantage of HT mixture is the muon sticking coefficient in $p t \mu$ molecule is very high. Therefore for future researches we must study on the reduction of muon sticking in HT mixture. Also we can deduce that, the TRIMUF measurements confirm the our theory predictions [6], [7].

\section{REFERENCES}

[1] F. C. Frank, Nature, vol. 160, pp. 525, 1947.

[2] L. W. Alvarez, H. Branda, F. S. Craford et al., Phys. Rev., vol. 105, pp. $1125,1957$.

[3] S. P. Merkuriev et al., "Proc Int Con on the Theory and Few Body and Quark-Hadronic Systems,” Dubna, vol. D4-87-692, p. 6, 1987.

[4] J. L. Friar, "Proc Int Con on the Theory and Few Body and QuarkHadronic Systems," Dubna, vol. D4-87-692, p. 70, 1987.

[5] H. Paetzgen, "Schieck," Few Body Systems, vol. 5, p. 171, 1988.

[6] Y. P. Averin, V. R. Bom, A. M. Demin et al., "Experimental result on muon catalyzed dt fusion in H/D/T mixture," Hyp.Int., vol. 138, p. 249 , 2001.

[7] A. M. Frolov and D. M. Wardlaw, "Bound state spectra of three-body muonic molecular ions," arXiv: [physics. atom-ph], vol. 1008, 2011.

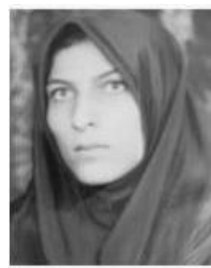

Seyeddeh Nasrin Hosseinimotlagh received her Ph.D and MS degree from the Physics Department of Shiraz University in Iran.

She is an assistant professor in Department of Physics, Shiraz branch Islamic Azad University, Shiraz, Iran. She has published in various journals. Department of Physics, Sciences College, Shiraz branch Islamic Azad University, Pardis, Sadra town, Shiraz, Iran. 


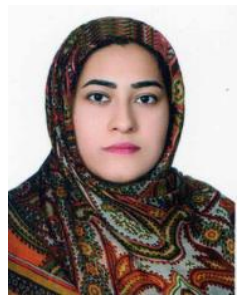

Sakineh Ghaderi received her B.S from Department of Physics, Shiraz branch Islamic Azad University, Shiraz, Iran. She is an university student in M.S in Neyshaboor university in solid state physics field.

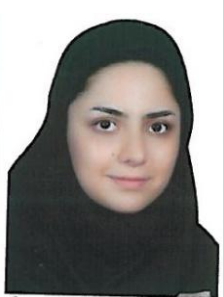

Fatemeh Mozafari koroosh received her B.S from Department of Physics, Shiraz branch Islamic Azad University, Shiraz, Iran. She is a university student in M.S in Dense Material Physics. She has published in various journals. Pardis Islamic Azad University of Shiraz, Department of Physics, Sciences College Shiraz branch Islamic Azad University, Pardis, Sadra town, Shiraz, Iran. 